\title{
Room-temperature operation of a unipolar nanodiode at terahertz frequencies
}

\author{
Claudio Balocco, ${ }^{1, a)}$ Shahrir R. Kasjoo, ${ }^{1}$ Xiaofeng F. Lu, ${ }_{1}^{1}$ Linqing Q. Zhang, ${ }^{1}$ \\ Yasaman Alimi, ${ }^{1}$ Stephan Winnerl, ${ }^{2}$ and Aimin M. Song ${ }^{1}$ \\ ${ }^{1}$ School of Electrical and Electronic Engineering, Photon Science Institute, University of Manchester, \\ Manchester M13 9PL, United Kingdom \\ ${ }^{2}$ Institute of Ion Beam Physics and Materials Research, Helmholtz-Zentrum Dresden-Rossendorf, \\ P.O. Box 510119, 01314 Dresden, Germany
}

(Received 29 March 2011; accepted 8 May 2011; published online 31 May 2011)

\begin{abstract}
We report on the room-temperature electrical rectification at $1.5 \mathrm{THz}$ of a unipolar nanodiode based on symmetry breaking in a nanochannel. The exploitation of its nonlinear diodelike characteristic and intrinsically low parasitic capacitance enables rectification at ultrahigh speed. The zero-voltage threshold and unique planar layout make the nanodiode suitable for building large arrays. This is the highest speed reported in nanorectifiers to date. (c) 2011 American Institute of Physics.
\end{abstract}

[doi:10.1063/1.3595414]

Interest in terahertz $(\mathrm{THz})$ technology $(1 \mathrm{THz}$ $\left.=10^{12} \mathrm{~Hz}\right)$ has been growing steadily given its outstanding potential and social impact. ${ }^{1-4}$ Several security applications have been envisaged, since the spectral signature of many explosives and chemical weapons are easily detected at $\mathrm{THz}$ frequencies. Furthermore, most clothes are transparent in this frequency range, making concealed weapons easier to be detected without the hazard of x-ray and improving the effectiveness of security checks in sensitive areas such as airports. So far, solid-state devices that are able to operate with a frequency bandwidth in the $\mathrm{THz}$ region have several major disadvantages, such as being commonly expensive, bulky, or requiring cryogenic cooling. This has remained as the main bottleneck to the development of compact, cost-effective $\mathrm{THz}$ systems. In the higher end of the $\mathrm{THz}$ spectrum, commonly referred to as far- and mid-infrared regime, compact solid-state, ultrafast diodes could potentially be used to harvest industrial waste heat which radiates at tens of THz. ${ }^{5}$ Similarly, a considerable part of the solar frequency spectrum also lies within the $\mathrm{THz}$ region, and a huge research effort with a large injection of capital, has been made in order to develop an effective way to convert this energy to usable dc electricity. Unfortunately, current solid-state solar technologies - such as photovoltaics - are unable to capture the energy radiated at $\mathrm{THz}$ frequencies due to the physical limitations of their band-to-band absorption working principle. ${ }^{6}$ Although some result has been achieved in exploring thermoelectric devices, their efficiency is generally too low to be considered as a viable technology for thermal or mid-infrared energy harvesting. ${ }^{7}$ In these regards, developing solid-state, room-temperature $\mathrm{THz}$ diodes could potentially benefit a few disciplines. Schottky diodes are currently the most well-developed devices capable of detecting $\mathrm{THz}$ radiation at room temperature, but at the expenses of a challenging fabrication. Their coupling with antennas and waveguide as well as the fabrication of large arrays also pose additional engineering issues. ${ }^{8}$ Resonant $\mathrm{THz}$ detectors based

\footnotetext{
${ }^{\text {a) }}$ Author to whom correspondence should be addressed. Electronic mail: claudio.balocco@manchester.ac.uk.
}

on the Dyakonov-Shur effect are also a viable alternative, but they require a tunable bias. ${ }^{9}$

Here we present the $\mathrm{THz}$ operation at room temperature of a device based on the rectenna paradigm. ${ }^{5,10,11}$ The radiation is collected by a planar $\mathrm{THz}$ microantenna, and rectified by a unipolar nanodiode, namely, a self-switching device (SSD), which is based on an asymmetric nanochannel (Fig. 1). ${ }^{12-14}$ The SSD is realized by tailoring the boundary of a narrow semiconductor channel to break its symmetry as shown in Fig. 1(a). An applied voltage $V$ not only changes the potential profile along the channel direction but also either widens or narrows the effective channel width, depending on its sign. With the device unbiased $(V=0)$, the effective channel width is almost pinched off by the surface states on
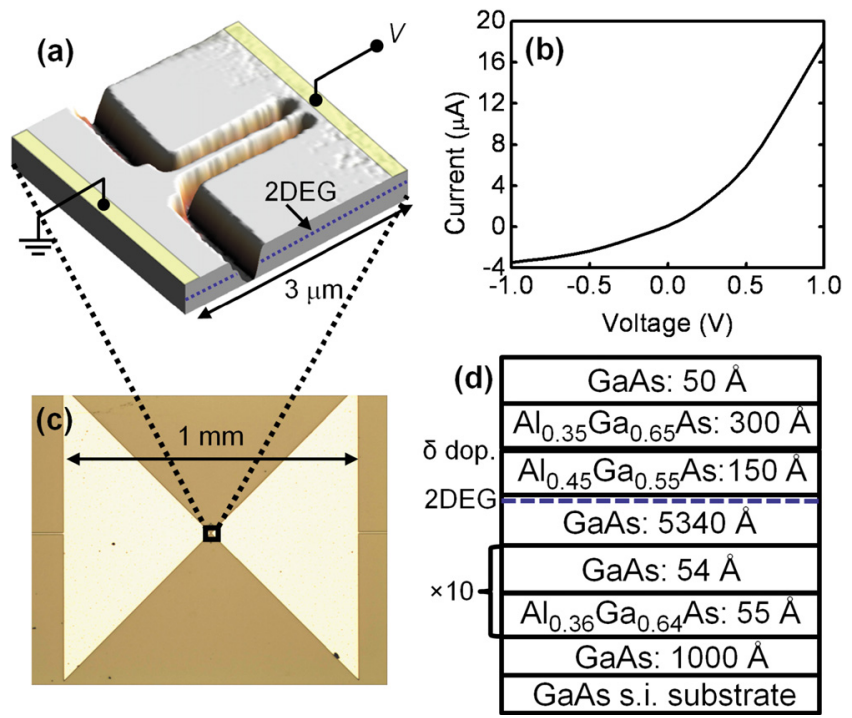

FIG. 1. (Color online) (a) Three-dimensional rendering of an atomic-force micrograph of an SSD, showing contact layout and etched trenches with the location of the 2DEG ( $z$ axis not in scale). The SSD channel is $1500 \mathrm{~nm}$ long and $210 \mathrm{~nm}$ wide, the trench width and depth are $110 \mathrm{~nm}$ and $130 \mathrm{~nm}$, respectively. (b) $I-V$ characteristic at room temperature of an array of four SSDs connected in parallel, fabricated in the gap of the bow-tie antenna. (c) Self-complementary bow-tie antenna used to couple the $\mathrm{THz}$ radiation to the SSDs. (d) Heterostructure utilized for the SSD fabrication. The location of the $2 \mathrm{DEG}$ is marked by the blue dashed line. 
the etched boundaries. A negative bias $(V<0)$ further depletes the channel whereas a positive bias $(V>0)$ counteracts the lateral depletion widening the effective width of the channel. This results in a strong nonlinear current-voltage $(I-V)$ characteristic, resembling that of a conventional diode, plotted in Fig. 1(b), but without using any doped junctions or any tunneling barriers. The SSD is entirely based on geometrical symmetry breaking at the nanoscale. It can, therefore, operate with a geometrically defined zero-voltage threshold which is independent of the material utilized. Moreover, the active area of the SSD is defined by a single high-resolution lithography step, thus avoiding the daunting alignment steps necessary for the fabrication of conventional vertical diodes. The single-step process makes the manufacturing of the nanodiodes compatible with cost-effective fabrication methods, such as nanoimprinting lithography (NIL). The device geometry can also benefit from multilevel NIL, ${ }^{15,16}$ which can be potentially utilized to define simultaneously both the antenna and the nanorectifier patterns, enabling denser structures at a lower cost.

The rectennas were fabricated out of a GaAs/AlGaAs wafer grown by molecular-beam epitaxy, sketched in Fig. $1(\mathrm{~d})$, in which free electrons were confined to a twodimensional electron gas (2DEG) in a quantum well located $50 \mathrm{~nm}$ below the surface. The carrier density and the electron mobility at a temperature of $300 \mathrm{~K}$ were $5.95 \times 10^{11} \mathrm{~cm}^{-2}$ and $\sim 7000 \mathrm{~cm}^{2} / \mathrm{V} \mathrm{s}$, respectively; at a temperature of $77 \mathrm{~K}$ were $5.55 \times 10^{11} \mathrm{~cm}^{-2}$ and $\sim 72000 \mathrm{~cm}^{2} / \mathrm{V} \mathrm{s}$, respectively, as determined by Hall measurements. The fabrication started with the definition of mesa structures to insulate adjacent devices by an $\mathrm{H}_{3} \mathrm{PO}_{4} / \mathrm{H}_{2} \mathrm{O}_{2} / \mathrm{H}_{2} \mathrm{O}$-based etch solution. Ohmic contacts were formed by thermal evaporation of a $50 \mathrm{~nm} \mathrm{Au} / \mathrm{Ge} / \mathrm{Ni}$ alloy followed by $200 \mathrm{~nm}$ of $\mathrm{Au}$ and annealing at $420{ }^{\circ} \mathrm{C}$. The $\mathrm{THz}$ antenna consists of a selfcomplementary bow-tie structure realized by thermal evaporation of a $200 \mathrm{~nm} \mathrm{Au}$ layer. Figure 1(c) shows an optical micrograph of the antenna. This design was chosen because of its simplicity and robustness to the variation in geometrical parameters as well as its reasonable wide band, and it is widely employed in $\mathrm{THz}$ systems. ${ }^{17,18}$ The selfcomplementary structure also provides real radiation impedance. However, the low antenna impedance of $72 \Omega$ (calculated as in Ref. 15) being mismatch to the SSDs' (approximately $150 \mathrm{k} \Omega$ ) prevented an effective power transfer, which resulted in suboptimal noise performance. The coupling to the Gaussian $\mathrm{THz}$ beam also suffered due to the antenna radiation pattern. ${ }^{17}$ More advanced antenna designs are likely to improve the overall performance. SSDs were exposed in polymethylmethacrylate (PMMA) by electronbeam lithography and subsequently transferred into the substrate by a $\mathrm{Br}_{2} / \mathrm{HBr} / \mathrm{HNO}_{3} / \mathrm{H}_{2} \mathrm{O}$-based wet etching, suitable for nanostructure fabrication. Four SSDs operating in parallel were placed in the antenna gap, in order to reduce the overall impedance and reducing the effect of the parasitic capacitance. The channels of the SSDs were $1500 \mathrm{~nm}$ long and $210 \mathrm{~nm}$ wide, the trench width and depth were $110 \mathrm{~nm}$ and $130 \mathrm{~nm}$, respectively, as determined by atomic-force micrographs. The design of the nanochannels was intentionally kept relatively wide, in order to provide a zero-threshold rectifier.

The $\mathrm{THz}$ beam was generated with a free-electron laser (FELBE at the Forschungszentrum Dresden-Rossendorf,

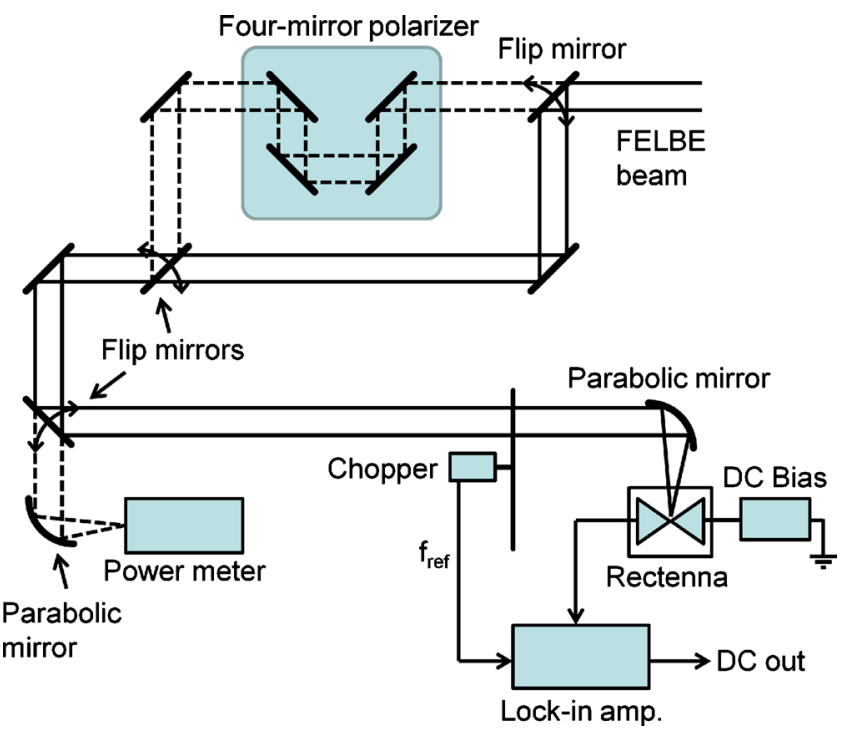

FIG. 2. (Color online) Diagram showing the measurement setup. The $\mathrm{THz}$ beam generated by FELBE was focused by a parabolic mirror onto the rectenna. The $\mathrm{THz}$ beam was stirred through a four-mirror polarizer to rotate the polarization of $90^{\circ}$ by a set of flip mirrors. A current source biased the rectenna and the rectified voltage was read out with a lock-in amplifier. A calibrated power meter and an aperture were also used to estimate the actual power density on the device.

Germany) and was focused on the rectenna by means of an off-axis parabolic mirror. The rectenna was mounted in a continuous-flow $\mathrm{He}$ cryostat with a diamond window transparent at $\mathrm{THz}$ frequencies. The polarization of the radiation was parallel to the antenna and could be rotated of $90^{\circ}$ by a four-mirror polarizer. The rectenna output was read out by a lock-in amplifier with the reference frequency provided by an optical chopper. A tunable dc bias could be applied to the rectenna trough a current source. Figure 2 shows the diagram of the measurement setup.

The rectified dc output as a function of the current bias at room temperature $(300 \mathrm{~K})$ at $1.5 \mathrm{THz}$ is shown in Fig. 3 . This is the highest speed reported at room temperature in nanorectifiers to date. The power delivered to the SSD antenna was estimated to be approximately $100 \mu \mathrm{W}$, taking into account the THz-beam diameter and the antenna effec-

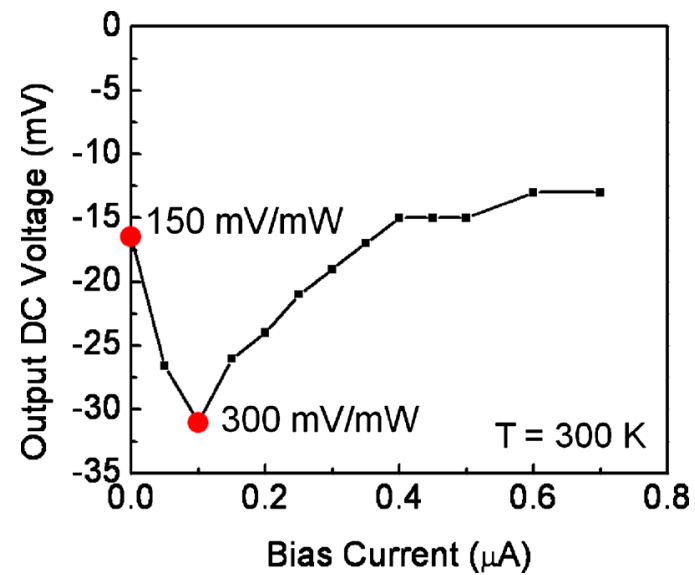

FIG. 3. (Color online) Rectified-voltage output from the rectenna at $1.5 \mathrm{THz}$ as a function of the SSD dc bias at room temperature $(300 \mathrm{~K})$. The power of the $\mathrm{THz}$ radiation was kept constant at $100 \mu \mathrm{W}$. Changing the dc allows for probing the different features and nonlinearity of the rectenna. The highest sensitivity was $300 \mathrm{mV} / \mathrm{mW}$ at a bias of $100 \mathrm{nA}$. 


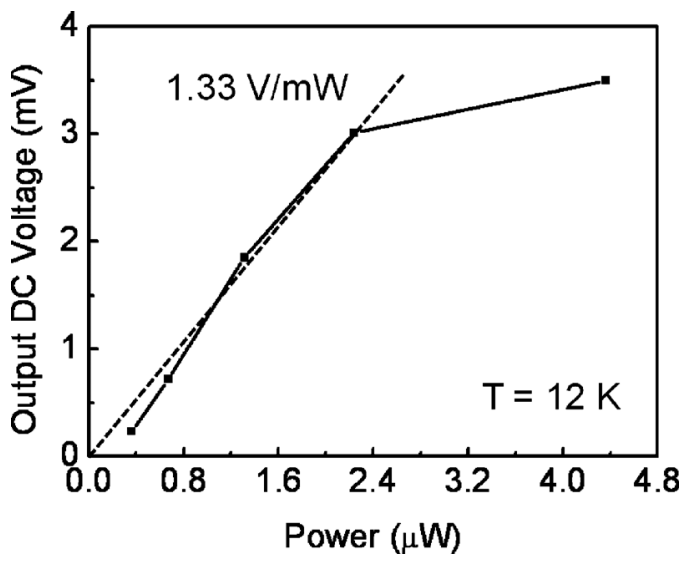

FIG. 4. Rectified-voltage output from the rectenna as a function of the power of the $\mathrm{THz}$ radiation at a temperature of $12 \mathrm{~K}$ with the rectenna unbiased. For power levels lower than $2 \mu \mathrm{W}$ the rectenna showed a linear response (square-law region)

tive area. At zero bias, the device detection efficiency was $150 \mathrm{mV} / \mathrm{mW}$, and the highest achieved was $300 \mathrm{mV} / \mathrm{mW}$ with a bias current of $100 \mathrm{nA}$. The dependence reflects the nonlinearity and differential resistance of the SSDs. The amount of de rectified by the SSDs is proportional to the nonlinearity of the $I-V$ characteristic (bowing coefficient), which increases with the current up to a certain bias level, and then slowly decreases due to the parasitic resistance in series with the SSDs. The dc is loaded on the differential resistance, which decreases as the bias current increases. ${ }^{19}$ Hence, the combination of both parameters as a function of the bias current results in the curve reported in Fig. 3. When the polarization was rotated of $90^{\circ}$ no significant signal was detected, indicating that the SSDs were only sensitive to the radiation received by the antenna, rather than environmental disturbances, such as local heating.

At zero bias and for frequencies high enough to neglect excess noises (typically above $1 \mathrm{MHz}$ ), the noise generated by the device is mainly due to thermal fluctuations in the channel (Johnson-Nyquist noise) with a power spectral density $S_{v}=4 k T R$, where $k$ is the Boltzmann's constant, $T$ the absolute temperature, and $R$ the channel resistance. ${ }^{20}$ The channel resistance at room temperature is approximately $150 \mathrm{k} \Omega$, yielding a voltage noise spectral density of $\sim 50 \mathrm{nV} / \mathrm{Hz}^{1 / 2}$. The room-temperature noise equivalent power at zero bias can then be estimated from the measured sensitivity of $150 \mathrm{mV} / \mathrm{mW}$, and it is approximately $330 \mathrm{pW} / \mathrm{Hz}^{1 / 2}$ at frequencies above $1 \mathrm{MHz}$, comparable to the values typically reported for Schottky diodes. ${ }^{8}$ Furthermore, the excess noise at lower frequencies, i.e., flicker noise and thermal noise can be strongly reduced by using larger arrays of SSDs connected in parallel (details to be published elsewhere). The rectenna performance, in term of noise and sensitivity, can also be improved by cooling. This allowed us to study the dependence of the rectified voltage as a function of the radiation power, as shown in Fig. 4. The rectenna was unbiased and at a temperature of $12 \mathrm{~K}$. The sensitivity was approximately $1.33 \mathrm{~V} / \mathrm{mW}$, as shown by the fit line, an order of magnitude higher than that measured at room temperature, which was ascribed to the much higher electron mobility of the 2DEG. The curve is only linear (or in the so-called square-law regime) for a power level lower than $2 \mu \mathrm{W}$, which indicates that the dynamic $I-V$ characteristic of the rectenna is well approximated by a parabolic curve. This is certainly true when the applied oscillating field can be considered as small signal, but by increasing the amplitude, the device is drawn in a relatively large-signal regime, which results in the observed sublinear dependence on the power.

Several devices were tested, all showing similar performances. Measurements in the microwave regime and numerical simulations (Silvaco) were also performed in order to validate the device operation principle. $1.5 \mathrm{THz}$ was the highest frequency we could utilize in our setup, which might not be upper operational limit of our devices. The optimization of several design parameters is also possible, such as antenna designs that are impedance-matched to the SSD and use of semiconductor with a higher electron mobility and carrier density, e.g., InAs and InSb. There is still large room for further increasing the speed of the SSD, and our current effort focuses on extending their operation toward the middle-infrared regime.

The authors wish to acknowledge the support of the EU FP7 projects under grant Agreements No. 226716 (ELISA) and 243845 (ROOTHz), UMIP, EPSRC Platform grant No. EP/E027261/1, and Photon Science Institute (PSI) of the University of Manchester. We are grateful to P. Michel and the FELBE team.

${ }^{1}$ B. B. Hu and M. C. Nuss, Opt. Lett. 20, 1716 (1995).

${ }^{2}$ M. Sherwin, Nature (London) 420, 131 (2002).

${ }^{3} \mathrm{Q}$. Chen and X. C. Zhang, in Ultrafast Lasers: Technology and Applications, edited by M. E. Fermann, A. Galvanauskas, and G. Sucha (Dekker, New York, 2001).

${ }^{4}$ P. de Bernardis, P. A. R. Ade, J. J. Bock, J. R. Bond, J. Borrill, A. Boscaleri, K. Coble, B. P. Crill, G. De Gasperis, P. C. Farese, P. G. Ferreira, K. Ganga, M. Giacometti, E. Hivon, V. V. Hristov, A. Iacoangeli, A. H. Jaffe, A. E. Lange, L. Martinis, S. Masi, P. V. Mason, P. D. Mauskopf, A. Melchiorri, L. Miglio, T. Montroy, C. B. Netterfield, E. Pascale, F. Piacentini, D. Pogosyan, S. Prunet, S. Rao, G. Romeo, J. E. Ruhl, F. Scaramuzzi, D. Sforna, and N. Vittorio, Nature (London) 404, 955 (2000).

${ }^{5}$ R. Corkish, M. A. Green, and T. Puzzer, Sol. Energy 73, 395 (2002).

${ }^{6}$ A. Luque and S. Hegedus, Handbook of Photovoltaic Science and Engineering (Wiley, Chichester, England, 2003).

${ }^{7}$ C. B. Vining, Nature Mater. 8, 83 (2009).

${ }^{8}$ F. Sizov and A. Rogalski, Prog. Quantum Electron. 34, 278 (2010).

${ }^{9}$ M. Dyakonov and M. Shur, IEEE Trans. Electron Devices 43, 380 (1996).

${ }^{10}$ D. L. Woolard, E. R. Brown, M. Pepper, and C. Kemp, Proc. IEEE 93, 1722 (2005)

${ }^{11}$ D. K. Kotter, S. D. Novack, W. D. Slafer, and P. J. Pinhero, J. Sol. Energy Eng. 132, 011014 (2010).

${ }^{12}$ A. M. Song, M. Missous, P. Omling, A. R. Peaker, L. Samuelson, and W. Seifert, Appl. Phys. Lett. 83, 1881 (2003).

${ }^{13}$ C. Balocco, A. M. Song, M. Åberg, A. Forchel, T. González, J. Mateos, I. Maximov, M. Missous, A. A. Rezazadeh, J. Saijets, L. Samuelson, D. Wallin, K. Williams, L. Worschech, and H. Q. Xu, Nano Lett. 5, 1423 (2005)

${ }^{14}$ C. Balocco, M. Halsall, N. Q. Vinh, and A. M. Song, J. Phys.: Condens. Matter 20, 384203 (2008).

${ }^{15}$ E. Lausecker, Y. Huang, T. Fromherz, J. C. Sturm, and S. Wagner, Appl Phys. Lett. 96, 263501 (2010).

${ }^{16}$ Y. Huang, B. Hekmatshoar, S. Wagner, and J. C. Sturm, IEEE Electron Device Lett. 29, 737 (2008)

${ }^{17}$ R. Compton, R. McPhedran, Z. Popovic, G. Rebeiz, P. Tong, and D. Rutledge, IEEE Trans. Antennas Propag. 35, 622 (1987).

${ }^{18}$ F. J. González and G. D. Boreman, Infrared Phys. Technol. 46, 418 (2005).

${ }^{19}$ D. M. Pozar, Microwave Engineering, 3rd ed. (Wiley, Hoboken, NJ, 2005).

${ }^{20}$ A. Van der Ziel, Noise: Sources, Characterization, Measurement (Prentice-Hall, Englewood Cliffs, NJ, 1970). 